\title{
Masculinidad y feminidad: ¿De qué estamos hablando?
}

\author{
Masculinity and Femininity: What are we Talking About?
}

\author{
Alí Roberto Chaves Jiménez' \\ Colegio Humanístico Costarricense \\ Universidad Nacional \\ Heredia, Costa Rica \\ archaves@una.ac.cr
}

Recibido 15 de marzo de 2012 • Corregido 04 de octubre de 2012 • Aceptado 07 de noviembre de 2012

Resumen. A continuación se presentan diversas ideas creadas alrededor del tema de la masculinidad y feminidad. El objetivo es esclarecer dichos conceptos desde un punto de vista crítico y de género sensitivo, en un lenguaje sencillo. El fin es que la información sea accesible a una mayor cantidad de personas; eso, sin menoscabar la importancia de transmitir las ideas desde un carácter académico y científico. Por tanto, se busca aclarar el carácter de ambos conceptos en la construcción de su identidad, principalmente para el hombre que cimienta su identidad a partir del rechazo de lo que es femenino. Se trata de mostrar cómo dichas construcciones son atravesadas por diversos mandatos sociales que se expresan según la cultura en la que se desarrollen; además, se plantea el reto de la educación para deconstruir estereotipos ligados a los géneros.

Palabras claves. Masculinidad, feminidad, patriarcado, género, derechos humanos, igualdad.

Abstract. By presenting some of the ideas constructed on masculinity and femininity, this paper aims at clarifying such concepts, in plain language, from a critical -and gender-sensitive- point of view, and make such information available to a wider public, without undermining, however, the importance of conveying ideas in academic and scientific terms, as well. Therefore, its purpose is to clarify the nature of both concepts in the construction of the individual's identity, particularly in the case of men whose identity is based on the rejection of femininity. This paper explains how such constructions are permeated by social mandates, which manifestation will depend on the culture. It also alerts us on the challenge of education in deconstructing gender-based stereotypes.

Keywords. Masculinity, femininity, patriarchy, gender, human rights and gender equality.

\footnotetext{
Bachiller en Orientación de la Universidad Nacional, egresado de Licenciatura en Orientación y de la Maestría en Derechos Humanos y Educación para la Paz de la Universidad Nacional. Miembro de la Federación de Estudiantes en el periodo 2006-2008, miembro propietario del Tribunal Universitario de Apelaciones de la Universidad Nacional, experiencia laboral como orientador y profesor de Psicología en el Holy Jesus High School, durante los meses de julio a diciembre del 2009 y, actualmente, desde enero del 2010, orientador en el Colegio Humanístico Costarricense de la Universidad Nacional.
} 
URL: http://www.una.ac.cr/educare

En la actualidad, el tema de la construcción de la masculinidad y feminidad nos concierne a todos y a todas por igual. Este ha marcado la historia y dado curso a un trato desigual entre hombres y mujeres. La distinción superior / inferior entre los géneros ha promovido en la cultura una percepción, desde el patriarcado, orientada a establecer una relación de poder, donde él manda y ella le sirve.

Hernández (2006), citando a Lamas, explica que el género es:

(...) una construcción simbólica e imaginaria que soporta los atributos asignados a las personas a partir de la interpretación cultural de su sexo: distinciones biológicas, físicas, económicas, sociales, psicológicas, eróticas, afectivas, jurídicas políticas y culturales impuestas. (p. 3)

Se ha marcado la diferencia entre ser hombre y ser mujer desde la educación que cada uno ha recibido desde su niñez. En esta educación se han generado construcciones tales como que la mujer es aquella persona que debe preocuparse por los demás antes que por ella misma y siempre debe estar dispuesta a servir y de la mejor manera. Por el contrario, el hombre tiene la oportunidad de decidir sobre las demás personas, puede exigir y equivocarse y, su estatus lo representa como un ser único, fuerte, admirable y correcto (INAMU, 2003).

(...) Por masculinidad se entiende una serie de significados de orden social que son dinámicos y se van construyendo de acuerdo a [sic] las necesidades y también por la interacción de "unos con otros". (Camacho, 2005, párr. 1)

Los hombres pretenden reafirmar su virilidad a través de actos que se alejan de lo femenino y se realizan durante todo el ciclo de vida. Con esto se han establecido una serie de actitudes que tienen como resultado la construcción de lo masculino como diferente de lo femenino y no a partir de atributos propios (Seidler, 2002).

El aprendizaje del ser masculino tiene como punto de partida el creerse con el control de todas las emociones y sentimientos hacia sí mismo, los demás y las situaciones en general, por lo que los varones desarrollan un espacio emocional limitado e inflexible, y llegan a confundir sus emociones con las expectativas que su grupo social tiene de ellos (Seidler, 2002).

Como se puede ver, la masculinidad, tradicionalmente, está unida a actitudes como la fuerza, la violencia, la agresividad y la idea de que es necesario estar probando y probándose continuamente que se es hombre (Seidler, 2002). 


\section{Definición de ideas básicas}

De acuerdo con López y Güida (2000), para comprender el significado de masculinidad y feminidad hay que tomar en consideración la construcción social, pues según la cultura se establecen conductas para una persona en función de su sexo biológico. Estos mandatos definen el género y el sentimiento de ser hombre o mujer, en construcciones que se dan en los primeros tres años de vida.

Los mismos autores refieren el feminismo como uno de los movimientos trascendentales de la historia, en la denominada ola del feminismo. Este tuvo un papel central en la visualización de la desigualdad de las mujeres como sujetos de derecho, de derechos sociales y civiles, del derecho a controlar su cuerpo -hoy llamados derechos sexuales y derechos reproductivos-, al placer sexual, a la anticoncepción, al aborto, a no ser discriminadas por su orientación sexual, entre otros.

El movimiento feminista ha sido promotor de los derechos de la mujer para que sean escuchadas y valoradas por sí mismas. Sin embargo, la cultura construye modos de ser mujer y de ser hombre, como menciona Simone de Bouvoir, citada por López y Güida, (2002), "(...) la mujer no nace, se hace" (p. 4) y extienden la misma idea hacia el hombre (...) el hombre no nace, sino que se hace" (p. 4), lo que evidencia que las construcciones de género refieren aspectos socioculturales, históricos, políticos, económicos y familiares, de ahí que, por ejemplo, se visualiza a la mujer con la pasividad y afectividad, y el hombre con la razón y la actividad.

López y Güida (2002) citan las ideas de pensadores como Platón, quien menciona que los hombres y las mujeres son capaces de realizar las mismas funciones, pero el hombre las realiza mejor -a excepción de las tareas vinculadas a lo doméstico-, por lo que se descalifica a la mujer en su importancia y se crea un rango, entre hombre y mujer, de superioridad e inferioridad.

Estos mismos autores confrontan esta idea de Platón, con el pensamiento de George Simmel, quien a inicios del siglo XX hace referencia a la posición centrada e intrínseca de que la mujer se diferencia del hombre, el cual adopta una posición en la que depende de la mujer para afirmarse como tal. Este autor, a diferencia de Platón, mantiene una posición a favor de la liberación de la mujer en una sociedad que se mueve en pro de la masculinidad.

Así mismo, Connell (2003) plantea el primer intento de construir una explicación científica de la masculinidad, la cual se dio desde la psicología moderna fundada a principios del siglo XX por Freud. Él ofreció la hipótesis de que todos los humanos tenían una constitución bisexual y que en cualquier persona coexistían corrientes masculinas y femeninas.

El mismo autor cita que Karen Horney, en 1932, en su libro El miedo a la mujer, expresa cómo el miedo a la madre se encuentra más afianzado y más reprimido que el miedo al padre castrante. De la misma forma establece el punto culminante de la crítica de la masculinidad en el psicoanálisis clásico; a partir de dos puntos importantes, los cuales son el grado en el cual la 
URL: http://www.una.ac.cr/educare

masculinidad adulta se construye a través de reacciones exageradas contra la feminidad, y la relación entre la formación de la masculinidad y la subordinación de las mujeres.

Kimmel (1997), citada por Valenzuela (2007) explica que

(...) la masculinidad está conformada por un conjunto de significados cambiantes, aunque recorridos por una constante: la construcción histórico-social de la virilidad que tiene lugar en oposición a las mujeres y a las minorías sexuales y raciales. Así, la masculinidad es ante todo la "huída de lo femenino" originada por la necesidad de distanciarse de la madre que representa la infancia desvalida, dependiente y castrada que el varón debe sepultar. La identidad masculina nace de la renuncia de lo femenino, no de la afirmación directa de lo masculino, lo cual deja a la identidad de género masculino tenue y frágil. (¿Qué es la masculinidad? párr. 2)

Por tanto, se establece que la masculinidad se construye a través de los procesos de socialización, y da como resultado una serie de masculinidades diversas. Estas resultan, según lugar de trabajo o condición socioeconómica, entre otros factores que pueden ser determinantes en los procesos de socialización masculina.

La conceptualización de hombre a nivel social se ha manejado de una manera autoritaria y apropiante ante los derechos de la mujer. La mujer se determina como un ser inferior, que se debe subordinar a los deseos del hombre, sin importar sus propios intereses.

Según el Instituto Nacional de las Mujeres (INAMU) (2003), la construcción de la identidad de género no se da en un tiempo determinado; se modela y se refuerza constantemente porque es una síntesis de las diferentes experiencias vividas; es un proceso continuo que tiene relación con el pasado, el presente y el futuro; es también la síntesis de las múltiples variables que condicionan o determinan la vida de las personas, como lo son la condición por las diferencias socioeconómicas, condición étnica, etaria, salud, lingüística y política, entre otras.

Esta manera de interpretar el mundo permite conocer las diferencias sexuales que caracterizan a las mujeres y a los hombres, y da paso a la desigualdad y a la inequidad entre estos dos grupos. Esta desigualdad e inequidad se expresan en diferencias marcadas como un puesto de trabajo, el desempeño profesional, la responsabilidad de la educación de los hijos e hijas, la obligación de proveer para las necesidades básicas en el hogar, entre otras; que se les asignan a unos y a otros solo por el hecho de ser mujeres u hombres.

Por esto, la sociedad le enseña a la mujer una serie de mandatos envueltos en una serie de mitos, los cuales dicen que ser mujer es ser dependiente, es decir, se sienten, se piensan y se representan en relación con las demás personas; y no en relación a sí mismas. Se les asignan las tareas de nutrir, comprender, proteger y sostener a otros. 
De acuerdo con el INAMU (2003), la omnipotencia es la que posibilita a las mujeres a encontrar excesivas estrategias para sostener a los otros, las estimula a estar alertas, ágiles y creativas para resolver lo que se presente.

El problema no está en tener tanta capacidad de trabajo, de creatividad y de iniciativa, el problema está en que:

- No se ha enseñado a las mujeres a dirigir todo ese potencial hacia su propio desarrollo personal.

- Cuando las mujeres se animan a pensar también en sus propias necesidades son severamente sancionadas y se les dice egoístas y desleales.

- Al resolver los problemas de los demás, ellos/as, no se verán obligados/as a aprender a resolverlos porque siempre habrá una mujer para actuar en el momento que lo necesiten (INAMU, 2003 p. 44).

También se relacionan con el miedo a sentirse rechazadas y abandonadas por los seres más cercanos a ellas. La culpa se puede presentar mediante el temor a realizar " $x$ " actividad y que esta no sea captada de manera positiva por otras personas, lo cual neutraliza cualquier intento de cambio.

Estas dos situaciones producen que la mujer se entregue a los demás y olvide su propio valor.

En cambio para los hombres la historia es diferente, pues se les educa como seres excepcionales con virtudes y aptitudes que los convierten en modelos a seguir. El ejemplo de esto es la perspectiva de Rousseau, citado por Subirats (1994), sobre la educación de los hombres y de las mujeres, ya que en la época de la llustración, este autor plantea que la educación de las mujeres no puede ser igual a la de los hombres, puesto que las mujeres nacieron para servirlos y el educarlas como si fueran hombres, es decir, el educarlas para que sean personas autónomas y conscientes de sí mismas, las dañaría para toda la vida. Se basa en la idea de que la mujer no aceptaría su papel en la sociedad como dependiente y subordinada.

Los hombres tienen la necesidad de demostrar que son hombres, requieren que otros los reconozcan. La sociedad patriarcal define al hombre como un ser potente con mandato y autoridad, con la mayoría de aprobación de sus actos, sean de carácter positivo o negativo, se le ha otorgado la dirección y el mandato como algo propio de su vida cotidiana, se le da el poder sobre el resto de las personas haciéndoles creer que son los dueños del poder y del conocimiento. 
URL: http://www.una.ac.cr/educare

El hombre requiere de una constante necesidad demostrativa que lo llenará de ego ante el resto de la sociedad, asociado a la realización de hazañas que lo satisfacen a nivel personal; también manifiesta la dependencia vital de otras personas que reconozcan sus hechos; el poder y la complicidad lo convierten en un ser con derecho a dar órdenes y si se equivoca, a no ser juzgado.

Sin embargo, esta discusión, plantea que no se puede encasillar la masculinidad o la feminidad en un estándar o una clasificación universal, ya que como se mencionan las diversas autoras y autores, y lo refuerza Otegui (1999):

(...) las identidades de género se deben estudiar como un continuo de formas simbólicas y prácticas sociales a través de las cuales las personas construyen su forma de ver el mundo, de actuar en el mismo, y de resituarse con relación a sí mismos y a su cuerpo (...). (p. 153)

Esto conlleva a revisar el planteamiento de una masculinidad y feminidad hegemónica, no negando su existencia, sino más bien, replanteando y agregando que históricamente se partió de la generalización, sin considerar factores como la edad, condición económica, etnia, religión, entre otras variables culturales que varían la forma en cómo se identifican genéricamente hombres y mujeres.

\section{Buscando el cambio}

Es importante reconocer la autonomía de cada individuo, sin distinción de género y, por lo tanto, merecedora de respeto. Así mismo, es necesario educar a los niños y niñas de manera imparcial, sin discriminación, de tal forma que se promueva su independencia y el desarrollo como seres humanos integrales.

Según la perspectiva de Lagarde (1997), tendríamos que aprender a constituir el yo y aprender a vivir desde nosotros; es decir, tener presente el crecer como personas, vivir desde una esfera de estar bien consigo mismo, con los demás, como el esperar un trato recíproco de los demás y para los demás. Por lo que cada persona, hombre y mujer, debe tener presente el potenciar su propio desarrollo para crecer como persona.

La influencia del medio que nos rodea, ya sea la iglesia, la política, la familia, la escuela y los medios de comunicación, entre otros, ha inculcado diversas ideas que estereotipan la diferenciación de los sexos. Por tanto, han designado roles en los cuales se subestima el género y se otorga un trato desigual con respecto a la masculinidad y a la feminidad.

La pregunta es: ¿por dónde se debe iniciar para poder llegar a construir una sociedad en la cual las personas se relacionen sin discriminarse entre sí? 
Existen diversas ideas sobre lo que sería la construcción de una igualdad sustantiva, ${ }^{2}$ por ejemplo, por medio de campañas publicitarias que busquen promover una sensibilización y concientización de género; sin embargo, esto tiene un alcance corto, ya que los medios de comunicación son los primeros en transmitir anuncios publicitarios donde lo primero que resalta es la discriminación: un ejemplo de ello es la constante mercantilización de la figura femenina. Asimismo, la música, como expresión de las diversas subculturas que se desarrollan en la sociedad, es también reproductora de ese modelo patriarcal, por medio de letras misóginas.

La educación es tal vez la principal reproductora de estereotipos sociales que se encuentran alrededor de la construcción identitaria de los niños como hombres y de las niñas como mujeres. A pesar de que se han desechado las ideas de Rousseau sobre la educación diferenciada para hombres y mujeres, los programas de educación carecen de una perspectiva de género que conlleve a una reflexión crítica del tema en las aulas; además, que la misma educación universitaria que reciben los maestros y las maestras adolece de un enfoque género sensitivo. Dentro de los planes de estudio se minimiza el tema de género a la simple incorporación de este como un eje transversal, y se reprime solo al hecho de escribir "los y las" para el caso que sea necesario.

Por tanto, si queremos realizar esa transformación real, sustantiva y trascendental, es necesario iniciar por una educación universitaria con un verdadero enfoque de género, ya que es allí donde se está formando a los futuros y futuras profesionales, quienes serán agentes multiplicadores dentro de la sociedad.

Un requisito fundamental es incorporar, además, en este proceso transformador, a toda la sociedad, lo cual incluye el repensar el modelo de sociedad, el pasar de un modelo capitalista a uno en el que el respeto de lo humano esté sobre la mercantilización, ya que al existir esta relación de subordinación (mercancías-ser humano) se da un detrimento de las relaciones sociales, con ello la imposibilidad de reconocernos y, consecuentemente, la posibilidad de crear relaciones humanas libres de discriminación se obstaculiza.

Es necesario buscar modelos alternativos en los cuales el valor de las personas esté por encima de cualquier otro aspecto que vaya a menoscabar las relaciones entre hombremujer, mujer-mujer y hombre-hombre; un modelo donde lo imperante sea el respeto de las

2 En cuanto al concepto de igualdad sustantiva, Facio (2009), explica que la igualdad se debe dar en dos planos, igualdad de jure y facto, con lo que es necesario establecer la igualdad no solo mediante la norma escrita, derecho positivo, punto que sea instaurado por medio del reconocimiento en las convenciones de derechos humanos, CEDAW y Belem do Para, de sus protocolos facultativos, entre otros, y de la incorporación de dicho marco legal en los diferentes países que ratificaron dichas convenciones, sino también, en la acción cotidiana, ya que a pesar de existir la norma, existen todavía grandes deficiencias en la promoción de dicha igualdad, pues continuamente la mujer es victima de violencia en todos los espacios sociales, la familia, el trabajo, grupos de pares, en la educación, entre otros y un reflejo de ello es el creciente número de femicidios que se dan, no solo en Costa Rica, sino a nivel mundial. 
URL: http://www.una.ac.cr/educare

diversidades y la aceptación de las mismas como medios para potencializar las relaciones de igualdad entre los géneros.

Resulta imperativo dejar atrás valores como los del capitalismo patriarcal, la mercantilización de los cuerpos, la competitividad extrema, en la que solo importa el ganar sin importar los medios, para pasar a un respeto de nosotras y nosotros mismos y de las demás personas y, partir del principio de cooperación, como medio para tender puentes que promuevan una sociedad sin discriminación. Esta es una tarea pendiente para todos los seres humanos que conforman la sociedad y se debe promover, desde la educación, lo que supone repensar esta como proceso transformador de las sensibilidades necesarias para convertir los demás entornos de la vida social.

\section{Referencias}

Camacho, S. (2005). Masculinidad, ¿el nuevo concepto? Enkidu. Recuperado de http://www. enkidumagazine.com/art/2005/280705/E 031 280705.htm

Connell, R. (2003). Masculinidades. México, DF.: Universidad Autónoma de México.

Facio, A. (2009). La igualdad substantiva. Un paradigma emergente de la ciencia jurídica. Recuperado de http://www.cidem-ac.org/PDFs/bibliovirtual/IGUALDAD/IGUALDAD\%20 SUSTANTIVA.\%20DRA.\%20ALDA\%20FACIO.pdf

Hernández, Y. (2006). Acerca del género como categoría analítica. Nómadas. Revista crítica de ciencias sociales y jurídicas, 13(1), 111-120. Recuperado de http://www.ucm.es/info/ nomadas/13/yhgarcia.pdf

Instituto Nacional de las Mujeres (INAMU). (2003). Módulo educativo psicología-secundaria: Aplicación de la visión de género a los programas de estudio del MEP. San José, Costa Rica: INAMU, MEP.

Lagarde, M. (1997). Identidad genérica y feminismo. Heredia, Costa Rica: Instituto de Estudios de la Mujer, Universidad Nacional.

López, A. y Güida, C. (2002). Aportes de los estudios de género en la conceptualización sobre masculinidad. Recuperado de www.inau.gub.uy/biblioteca/sexu4.doc

Otegui, R. (1999). La construcción social de las masculinidades. Política y sociedad, 32, 151-160. Recuperado de http://revistas.ucm.es/index.php/POSO/article/view/ $\underline{\text { POSO9999330151A/24698 }}$

Seidler, V. (2002). Transformando masculinidades: El trabajo, la familia, y la cultura. En EMAKUNDE (Ed.), Congreso Internacional los hombres ante el nuevo orden social (pp. 19-28). Vitoria-Gasteiz, 
España: EMAKUNDE. Recuperado de http://www.emakunde.euskadi.net/u72-publicac/es/ contenidos/informacion/pub jornadas/es emakunde/adjuntos/congreso2002 \%20es.pdf

Subirats, M. (septiembre-diciembre, 1994). Conquistar la igualdad: La coeducación hoy. Revista Iberoamericana de Educación, 6, 49-78.

Valenzuela, P. (3 de agosto de 2007). Masculinidad y relaciones de poder entre los hombres [Mensaje en un blog]. Recuperado de http://logos.psykhe.org/2007/08/masculinidad-y-relacionesde-poder.html

\section{Cómo citar este artículo, según APA:}

Chaves, A. R. (2012). Masculinidad y feminidad: ¿De qué estamos hablando? Revista Electrónica Educare, 16 (Especial), 5-13. Consultado de http://www.revistas.una.ac.cr/index.php/EDUCARE/issue/view/418

Nota: Para citar este artículo en otros sistemas puede consultar el hipervínculo "Como citar el artículo" en la barra derecha de nuestro sitio web: http://www.revistas.una.ac.cr/index.php/ EDUCARE/index 